\title{
Periodic averaging method for impulsive stochastic dynamical systems driven by fractional Brownian motion under non-Lipschitz condition
}

\author{
Anas Dheyab Khalaf1*, Mahmoud Abouagwa² and Xiangjun Wang ${ }^{1}$
}

"Correspondence:

anasdheyab@hust.edu.cn

${ }^{1}$ School of Mathematics and

Statistics, Huazhong University of Science and Technology, Wuhan, P.R. China

Full list of author information is available at the end of the article

\begin{abstract}
This paper presents the periodic averaging principle for impulsive stochastic dynamical systems driven by fractional Brownian motion (fBm). Under non-Lipschitz condition, we prove that the solutions to impulsive stochastic differential equations (ISDEs) with fBm can be approximated by the solutions to averaged SDEs without impulses both in the sense of mean square and probability. Finally, an example is provided to illustrate the theoretical results.
\end{abstract}

Keywords: Periodic averaging technique; Non-Lipschitz condition; Fractional Brownian motion; Impulsive dynamical systems; Stochastic differential equations

\section{Introduction}

In the past years, stochastic dynamical systems driven by $\mathrm{fBm}$ have became an active area of investigation due to their applications in telecommunications networks, finance markets, biology, and other fields [1-6]. The impulsive effects exist widely in many evolution processes in which states are changed abruptly at certain moments of time. Consequently, the impulsive differential equations have a wide range of applications in numerous branches of sciences such as finance, economics, medicine, biology, electronics, and telecommunications (see [7-10]).

On the other hand, it is well known that the averaging technique represents a good mathematical tool that approximates complicated time varying differential equations to autonomous differential equations. Since Krylov and Bogolyubov [11] put forward the cornerstone of the averaging principles for deterministic dynamical systems, averaging method has received considerable attention, and it has been found available and useful for exploring dynamical systems in many fields [12-16]. Up to now, there have been some works about stochastic averaging for dynamic problems with Gaussian random perturbation [17-19], Poisson noise [20, 21], Lévy motion [22-25], G-Brownian motion [26, 27], and $\mathrm{fBm}$ [28-31]. So far, no previous study has employed the periodic averaging technique to impulsive stochastic dynamical systems with $\mathrm{fBm}$. Therefore, we make an attempt to

(c) The Author(s) 2019. This article is licensed under a Creative Commons Attribution 4.0 International License, which permits use sharing, adaptation, distribution and reproduction in any medium or format, as long as you give appropriate credit to the original author(s) and the source, provide a link to the Creative Commons licence, and indicate if changes were made. The images or other third party material in this article are included in the article's Creative Commons licence, unless indicated otherwise in a credit line to the material. If material is not included in the article's Creative Commons licence and your intended use is not permitted by statutory regulation or exceeds the permitted use, you will need to obtain permission directly from the copyright holder. To view a copy of this licence, visit http://creativecommons.org/licenses/by/4.0/. 
establish the periodic averaging principle to ISDEs with $\mathrm{fBm}$, which allows the averaged systems without impulses to replace the original ISDEs both in mean square sense and probability.

We consider a class of ISDEs with fBm of the form

$$
\begin{aligned}
& d x(t)=a(t, x(t)) d t+b(t, x(t)) d W^{H}(t), \quad t \neq t_{j}, \\
& \triangle x\left(t_{j}\right)=I_{j}\left(x\left(t_{j}^{-}\right)\right), \quad t=t_{j}, j \in \mathbb{N}, \\
& x(0)=x_{0},
\end{aligned}
$$

where $\Delta x\left(t_{j}\right)$ denotes the jump of $x$ at $t=t_{j}$, for $0 \leq t \leq T<\infty$, and $\Delta x\left(t_{j}\right)=x\left(t_{j}^{+}\right)-x\left(t_{j}^{-}\right)$, such that $x\left(t_{j}^{+}\right)=\lim _{t \rightarrow t_{j}^{+}} x(t)$ and $x\left(t_{j}^{-}\right)=\lim _{t \rightarrow t_{j}^{-}} x(t) . x_{0}$ represents the initial data of the system with $E\left|x_{0}\right|^{2}<\infty$. The process $W^{H}(t)$ is fBm with Hurst index $H \in\left(\frac{1}{2}, 1\right)$ defined on the filtered probability space $\left(\Omega, \mathcal{F},\left\{\mathcal{F}_{t}\right\}_{t \geq 0}, P\right)$. The coefficients $a(t, x(t)):[0, T] \times R^{n} \rightarrow R^{n}$ and $b(t, x(t)):[0, T] \times R^{n} \rightarrow R^{n \times m}$ are measurable functions.

The outline of this manuscript is as follows. In Sect. 2, we provide some background about stochastic integral with respect to $\mathrm{fBm}$. Section 3 is devoted to establishing the stochastic periodic averaging approach to Eq. (1) under non-Lipschitz condition. Finally, an example is presented to demonstrate the theoretical results in Sect. 4.

\section{Framework}

In this section, we introduce some basic notions and preliminaries on path-wise integrals with respect to $\mathrm{fBm}$, and for more detailed discussion, we refer the reader to [6, 32-35].

Let $\left(\Omega, \mathcal{F},\left\{\mathcal{F}_{t}\right\}_{t \geq 0}, P\right)$ be a complete probability space equipped with a natural filtration $\left\{\mathcal{F}_{t}\right\}_{t \geq 0}$, where $\mathcal{F}_{t}$ is the $\sigma$-algebra generated by $\left\{W^{H}(t), t \in[0, T]\right\}$ and $\mathcal{F}_{0}$ contains all $P$-null sets.

Definition 2.1 The process $\left\{W^{H}(t), 1 / 2<H<1\right\}$ is said to be a centered self-similar fBm if the following properties are satisfied:

- $W^{H}(0)=0$,

- $E\left[W^{H}(t)\right]=0, t \in[0, T]$

- $E\left[W^{H}(t) W^{H}(s)\right]=\frac{1}{2}\left(|t|^{2 H}+|s|^{2 H}-|t-s|^{2 H}\right), t, s \in[0, T]$.

Next, for the convenience of readers, we provide some basic properties on path-wise integrals. Firstly, we introduce the function $\varphi: \mathbb{R}_{+} \times \mathbb{R}_{+} \rightarrow \mathbb{R}_{+}$defined as

$$
\varphi(t, s)=H(2 H-1)|t-s|^{2 H-2}, \quad t, s \in \mathbb{R}_{+},
$$

where $H \in\left(\frac{1}{2}, 1\right)$. Let $f: \mathbb{R}_{+} \rightarrow \mathbb{R}_{+}$be a Borel measurable function and define the space

$$
L_{\varphi}^{2}\left(\mathbb{R}_{+}\right)=\left\{f:\|f\|_{\varphi}^{2}=\int_{\mathbb{R}_{+}} \int_{\mathbb{R}_{+}} f(t) f(s) \varphi(t, s) d s d t<\infty\right\}
$$

which becomes a separable Hilbert space under the inner product

$$
\left\langle f_{1}, f_{2}\right\rangle_{\varphi}=\int_{\mathbb{R}_{+}} \int_{\mathbb{R}_{+}} f_{1}(t) f_{2}(s) \varphi(t, s) d s d t, \quad f_{1}, f_{2} \in L_{\varphi}^{2}\left(\mathbb{R}_{+}\right) .
$$


Now, consider the set $\mathcal{E}$ of smooth and cylindrical random variables of the form

$$
F(\omega)=g\left(\int_{0}^{T} \psi_{1}(t) d W^{H}(t), \ldots, \int_{0}^{T} \psi_{n}(t) d W^{H}(t)\right)
$$

where $n \geq 1$ and $g \in \mathcal{C}_{b}^{\infty}\left(\mathbb{R}^{n}\right)$ (i.e., $g$ and its partial derivatives are bounded). Moreover, let $\mathcal{H}$ be the family of measurable functions such that, for $\psi_{i} \in \mathcal{H}, i=1, \ldots, n, n \in \mathbb{N}$, we have $\left\langle\psi_{i}, \psi_{j}\right\rangle_{\varphi}=\delta_{i j}$ and $\|\psi\|_{\varphi}^{2}<\infty$. The elements of $\mathcal{H}$ may not be functions but distributions of negative order. Thanks to this reason, it is convenient to introduce the space $|\mathcal{H}|$ of measurable functions $h$ on $[0, T]$ satisfying

$$
\|h\|_{|\mathcal{H}|}^{2}=\int_{0}^{T} \int_{0}^{T}|h(t)||h(s)| \varphi(t, s) d s d t<\infty
$$

and it is easy to show that $|\mathcal{H}|$ is a Banach space under the norm $\|\cdot\|_{|\mathcal{H}|}$.

Definition 2.2 The Malliavin derivative $D_{t}^{H}$ of a smooth and cylindrical random variable $F$ is defined as an $\mathcal{H}$-valued random variable such that

$$
D_{t}^{H} F=\sum_{i=1}^{n} \frac{\partial g}{\partial x_{i}}\left(\int_{0}^{T} \psi_{1}(t) d W^{H}(t), \ldots, \int_{0}^{T} \psi_{n}(t) d W^{H}(t)\right) \psi_{i}(t)
$$

hence, $D_{t}^{H}$ represents a closable operator, so that $D_{t}^{H}: L^{p}(\Omega) \mapsto L^{p}(\Omega, \mathcal{H}), p \geq 1$. The iteration of Malliavin derivative is denoted by $D_{t}^{H, k}, k \geq 1$. For any $p \geq 1$, the Sobolev space $\mathbb{D}^{k, p}$ represents the closer of $\mathcal{E}$ with respect to the norm

$$
\|F\|_{k, p}^{p}=E|F|^{p}+E \sum_{i=1}^{k}\left\|D_{t}^{H, i} F\right\|_{\mathcal{H} \otimes i}^{p},
$$

where $\otimes$ denotes the tensor product.

Similarly, for a Hilbert space $U$, we denote by $\mathbb{D}^{k, p}(U)$ the corresponding Sobolev space of $U$-valued random variables, and for $p>0$, we denote by $\mathbb{D}^{1, p}(|\mathcal{H}|)$ the subspace of $\mathbb{D}^{1, p}(\mathcal{H})$ formed by the elements $h$ of $|\mathcal{H}|$. According to [6], we introduce $\varphi$-derivative of $F$ as follows:

$$
D_{t}^{\varphi} F=\int_{\mathbb{R}_{+}} \varphi(t, s) D_{s}^{H} F d s .
$$

Definition 2.3 The space $\mathcal{L}_{\varphi}[0, T]$ of integrals is defined as the family of stochastic processes $V(t)$ on $[0, T]$ such that $E\|V(t)\|_{\varphi}^{2}<\infty, V(t)$ is $\varphi$-differentiable, the trace of the derivative $D_{s}^{\varphi} V(t)$ exists, and for $t, s \in[0, T]$,

$$
E\left[\int_{0}^{T} \int_{0}^{T}\left|D_{t}^{\varphi} V(s)\right|^{2} d s d t\right]<\infty .
$$

In addition, for each sequence of partitions $\left(\pi_{n}, n \in \mathbb{N}\right)$ with $\left|\pi_{n}\right| \rightarrow 0$ as $n \rightarrow \infty$, the following are satisfied:

$$
\sum_{i=0}^{n-1} E\left[\int_{t_{i}^{(n)}}^{t_{i+1}^{(n)}} \int_{t_{j}^{(n)}}^{t_{j+1}^{(n)}}\left|D_{s}^{\varphi} V^{\pi}\left(t_{i}^{(n)}\right) D_{t}^{\varphi} V^{\pi}\left(t_{j}^{(n)}\right)-D_{s}^{\varphi} V(t) D_{t}^{\varphi} V(s)\right|^{2} d s d t\right] \rightarrow 0
$$


and

$$
E\left\|V^{\pi}-V\right\|_{\varphi}^{2} \rightarrow 0
$$

as $n$ tends to infinity, where $\pi_{n}=t_{0}^{(n)}<t_{1}^{(n)}<\cdots<t_{n-1}^{(n)}<t_{n}^{(n)}=T,|\pi|:=\max _{i}\left(t_{i+1}-t_{i}\right)$ and $V^{\pi}=V_{t_{i}}$.

Now, define the space $\mathbb{H}_{\varphi}^{1,2}$, which represents the intersection of the spaces $\mathbb{D}^{1,2}(|\mathcal{H}|)$ and $\mathcal{L}_{\varphi}[0, T]$, such that $\mathbb{H}_{\varphi}^{1,2}=\mathbb{D}^{1,2}(|\mathcal{H}|) \cap \mathcal{L}_{\varphi}[0, T]$.

Definition 2.4 Let $V(t)$ be a stochastic process with integrable trajectories.

- The symmetric integral of $V(t)$ with respect to $W^{H}(t)$ is defined as follows:

$$
\lim _{\epsilon \rightarrow 0} \frac{1}{2 \epsilon} \int_{0}^{T} V(s)\left[W^{H}(s+\epsilon)-W^{H}(s-\epsilon)\right] d s,
$$

provided that the limit exists in probability, the symmetric integral is denoted by

$$
\int_{0}^{T} V(s) d^{\circ} W^{H}(s)
$$

- The forward integral of $V(t)$ with respect to $W^{H}(t)$ is defined as follows:

$$
\lim _{\epsilon \rightarrow 0} \frac{1}{\epsilon} \int_{0}^{T} V(s)\left[\frac{W^{H}(s+\epsilon)-W^{H}(s)}{\epsilon}\right] d s,
$$

provided that the limit exists in probability, the forward integral is denoted by

$$
\int_{0}^{T} V(s) d^{-} W^{H}(s)
$$

- The backward integral of $V(t)$ with respect to $W^{H}(t)$ is defined as follows:

$$
\lim _{\epsilon \rightarrow 0} \frac{1}{\epsilon} \int_{0}^{T} V(s)\left[\frac{W^{H}(s-\epsilon)-W^{H}(s)}{\epsilon}\right] d s,
$$

provided that the limit exists in probability, the backward integral is denoted by

$$
\int_{0}^{T} V(s) d^{+} W^{H}(s) .
$$

In order to establish our results, we need to introduce some lemmas. The next lemma follows (Remark 1 in [35]) and (Proposition 6.2.3 in [6]).

Lemma 2.5 If the stochastic process $V(t)$ satisfies

$$
\int_{0}^{T} \int_{0}^{T}\left|D_{s}^{H} V(t)\right||t-s|^{2 H-2} d s d t<\infty, \quad V \in \mathbb{D}^{1,2}(|\mathcal{H}|),
$$

then the symmetric integral coincides with the forward and backward integrals. 
Since $\mathrm{fBm}$ is neither semi-martingale nor Markov process, we definitely lost the use of Burkholder-Davis-Gundy inequality and Ito-isometry. Therefore, there is a pressing need to use the following two lemmas from [6] and [28].

Lemma 2.6 If $V(t)$ is a stochastic process on $\mathbb{H}_{\varphi}^{1,2}$, then the symmetric integral is well defined and

$$
\int_{0}^{T} V(s) d^{\circ} W^{H}(s)=\int_{0}^{T} V(s) \diamond d W^{H}(s)+\int_{0}^{T} D_{s}^{\varphi} V(s) d s
$$

where $\diamond$ denotes the Wick product.

We note that the forward and backward integrals are also well defined. Hence, by Lemma 2.5, the forward and backward integrals coincide with the symmetric integral under the condition of Lemma 2.6.

Lemma 2.7 Let $W^{H}(t)$ be fBm with Hurst index $H \in\left(\frac{1}{2}, 1\right)$ and $V(t)$ be a stochastic process in $\mathbb{H}_{\varphi}^{1,2}$, then, for $0 \leq T<\infty$, there exists a constant $C>0$ such that

$$
E\left|\int_{0}^{T} V(s) d^{\circ} W^{H}(s)\right|^{2} \leq 2 H T^{2 H-1} E \int_{0}^{T}|V(s)|^{2} d s+4 C T^{2} .
$$

The following requisite lemma is taken from [36].

Lemma 2.8 Let $T>0, x_{0} \geq 0$, and $x(t), y(t)$ be two continuous functions on $[0, T]$. Assume that $\kappa: \mathbb{R}_{+} \rightarrow \mathbb{R}_{+}$is a concave continuous nondecreasing function such that $\kappa(v)>0$ for $v>0$. If we have

$$
x(t) \leq x_{0}+\int_{0}^{t} y(s) \kappa(x(s)) d s \quad \forall t \in[0, T]
$$

then

$$
x(t) \leq G^{-1}\left(G\left(x_{0}\right)+\int_{0}^{t} y(s) d s\right) \quad \forall t \in[0, T]
$$

where $\left(G\left(x_{0}\right)+\int_{0}^{t} y(s) d s\right) \in \operatorname{Dom}\left(G^{-1}\right), G(v)=\int_{0}^{v} \frac{d s}{\kappa(s)} d s, v>0$. Moreover, if $x_{0}=0$ and $\int_{0^{+}} \frac{d s}{\kappa(s)} d s=\infty$, then $x(t)=0$ for all $t \in[0, T]$.

Throughout this paper, the following assumptions are imposed.

Assumption A For all $x, y \in \mathbb{R}^{n}, t \in[0, T]$, and $a(t, \cdot), b(t, \cdot) \in \mathbb{H}_{\varphi}^{1,2}$, there exists a function $\kappa(\cdot)$ such that

$$
|a(t, x)-a(t, y)|^{2}+|b(t, x)-b(t, y)|^{2}+\left|D_{t}^{\varphi}(b(t, x)-b(t, y))\right|^{2} \leq \kappa\left(|x-y|^{2}\right),
$$

where $\kappa(\cdot)$ is a concave continuous nondecreasing function such that $\kappa(0)=0$ and

$$
\int_{0^{+}} \frac{1}{\kappa(x)} d x=\infty
$$


Moreover, since $\kappa(\cdot)$ is a concave continuous nondecreasing function, then there must exist two constants $\lambda_{1}>0$ and $\lambda_{2}>0$ such that

$$
\kappa(x) \leq \lambda_{1} x+\lambda_{2} .
$$

Remark 2.9 In view of Assumption A, we can see clearly, for a special case, if $\kappa(|x|)=K|x|$, then the Lipschitz condition is recovered. Therefore, Assumption A is much weaker than the usual Lipschitz condition.

Next, according to Lemma 3.1 in [37], the solution of impulsive stochastic dynamical system (1) can be given by the following integral equation:

$$
x(t)=x_{0}+\int_{0}^{t} a(s, x(s)) d s+\int_{0}^{t} b(s, x(s)) d^{\circ} W^{H}(s)+\sum_{0<t_{j}<t} I_{j}\left(x\left(t_{j}\right)\right) .
$$

Now, consider the standard ISDE with $\mathrm{fBm}$

$$
x_{\epsilon}(t)=x_{0}+\epsilon^{2 H} \int_{0}^{t} a\left(s, x_{\epsilon}(s)\right) d s+\epsilon^{H} \int_{0}^{t} b\left(s, x_{\epsilon}(s)\right) d^{\circ} W^{H}(s)+\epsilon^{H} \sum_{0<t_{j}<t} I_{j}\left(x_{\epsilon}\left(t_{j}\right)\right),
$$

where $\epsilon \in\left(0, \epsilon_{0}\right]$ is a positive small parameter and $\epsilon_{0}$ is a fixed number. Moreover, the averaged SDE of the standard ISDE (3) is

$$
z_{\epsilon}(t)=x_{0}+\epsilon^{2 H} \int_{0}^{t}\left[\bar{a}\left(z_{\epsilon}(s)\right)+\bar{I}\left(z_{\epsilon}(s)\right)\right] d s+\epsilon^{H} \int_{0}^{t} \bar{b}\left(z_{\epsilon}(s)\right) d^{\circ} W^{H}(s),
$$

where the functions $\bar{a}(x): \mathbb{R}^{n} \rightarrow \mathbb{R}^{n}, \bar{b}(x): \mathbb{R}^{n} \rightarrow \mathbb{R}^{n}$ and $\bar{I}(x): \mathbb{R}^{n} \rightarrow \mathbb{R}^{n}$ are measurable functions satisfying

$$
\begin{aligned}
& \bar{a}(x)=\frac{1}{T} \int_{0}^{T} a(t, x) d t, \\
& \bar{b}(x)=\frac{1}{T} \int_{0}^{T} b(t, x) d t, \\
& \bar{I}(x)=\frac{1}{T} \sum_{j=1}^{k} I_{j}(x) .
\end{aligned}
$$

Assumption B For any $x, y \in \mathbb{R}^{n}$, there exist positive constants $N_{1}$ and $N_{2}$ such that

$$
\left|I_{j}(x)\right|^{2} \leq N_{1}, \quad\left|I_{j}(x)-I_{j}(y)\right|^{2} \leq N_{2}|x-y|^{2} .
$$

Assumption C For all $t \in[0, T], x \in \mathbb{R}^{n}$, the coefficients of Eq. (3) and Eq. (4) are bounded. Then there exists a positive constant $M$ such that

$$
|a(t, x)|^{2} \leq M, \quad|b(t, x)|^{2} \leq M, \quad|\bar{a}(x)|^{2} \leq M, \quad|\bar{b}(x)|^{2} \leq M
$$

Now, the existence and uniqueness result for Eq. (2) is given by the following theorem.

Theorem 2.10 Assume that Assumptions A-C are satisfied. Then, for every initial value $x_{0} \in \mathbb{R}^{n}$, there exists a unique solution $x(t)$ to Eq. (2) on $[0, T]$. 
Proof The proof is a special case of the proof of Theorem 3.1 in Abouagwa et al. [38] and easy to be derived. So, we omit the proof here.

\section{Periodic averaging principle}

In this section, we study the periodic averaging principle of ISDEs driven by $\mathrm{fBm}$ under non-Lipschitz condition.

In order to provide the periodic averaging results, we assume that the functions $a$ and $b$ are T-periodic in the first argument and the impulses $I_{j}$ are periodic in the sense that there exist $k \in \mathbb{N}$ such that $0 \leq t_{1}<t_{2}<\cdots<t_{k}<T$, and for every $j>k$, we have $t_{j}=t_{j-k}+T$, $I_{j}=I_{j-k}$.

Following Theorem 3.6 in Mao et al. [39], we now establish our main result which is used for revealing the relationship between the processes $x_{\epsilon}(t)$ and $z_{\epsilon}(t)$.

Theorem 3.1 Consider standard ISDE (3) and averaging SDE (4) if Assumptions A-C hold. Then, for $T>0$, the following equality is satisfied:

$$
\lim _{\epsilon \rightarrow 0} E\left|x_{\epsilon}(t)-z_{\epsilon}(t)\right|^{2}=0
$$

Proof From Eqs. (3) and (4), taking expectation and employing the basic inequality $\mid a+$ $b+\left.c\right|^{2} \leq 3|a|^{2}+3|b|^{2}+3|c|^{2}$, we obtain

$$
\begin{aligned}
E\left|x_{\epsilon}(t)-z_{\epsilon}(t)\right|^{2} \leq & 3 \epsilon^{4 H} E\left|\int_{0}^{t} a\left(s, x_{\epsilon}(s)\right)-\bar{a}\left(z_{\epsilon}(s)\right) d s\right|^{2} \\
& +3 \epsilon^{2 H} E\left|\int_{0}^{t} b\left(s, x_{\epsilon}(s)\right)-\bar{b}\left(z_{\epsilon}(s)\right) d^{\circ} W^{H}(s)\right|^{2} \\
& +3 \epsilon^{2 H} E\left|\sum_{j=1}^{\infty} I_{j}\left(x_{\epsilon}\left(t_{j}\right)\right)-\int_{0}^{t} \bar{I}\left(z_{\epsilon}(s)\right) d s\right|^{2} \\
= & \sum_{l=1}^{3} Q_{l} .
\end{aligned}
$$

Starting with the first term $Q_{1}$, we have

$$
\begin{aligned}
Q_{1} & \leq 6 \epsilon^{4 H} E\left|\int_{0}^{t} a\left(s, x_{\epsilon}(s)\right)-a\left(s, z_{\epsilon}(s)\right) d s\right|^{2}+6 \epsilon^{4 H} E\left|\int_{0}^{t} a\left(s, z_{\epsilon}(s)\right)-\bar{a}\left(z_{\epsilon}(s)\right) d s\right|^{2} \\
& =Q_{11}+Q_{12} .
\end{aligned}
$$

For $Q_{11}$, by applying the Cauchy-Schwarz inequality, Jensen's inequality, and Assumption $\mathrm{A}$, one can get

$$
\begin{aligned}
Q_{11} & \leq 6 \epsilon^{4 H} E\left(t \int_{0}^{t}\left|a\left(s, x_{\epsilon}(s)\right)-a\left(s, z_{\epsilon}(s)\right)\right|^{2} d s\right) \\
& \leq 6 \epsilon^{4 H} \sup _{0 \leq t \leq u} t\left(E \int_{0}^{t} \kappa\left(\left|x_{\epsilon}(s)-z_{\epsilon}(s)\right|^{2}\right) d s\right) \\
& \leq 6 \epsilon^{4 H} u \int_{0}^{t} \kappa\left(E\left|x_{\epsilon}(s)-z_{\epsilon}(s)\right|^{2}\right) d s .
\end{aligned}
$$


Now, to deal with $Q_{12}$, let $m$ be the largest positive integer such that $m T \leq t$. Then, for every $i=1, \ldots, m$,

$$
\begin{aligned}
Q_{12} \leq & 12 \epsilon^{4 H} E\left|\sum_{i=1}^{m} \int_{(i-1) T}^{i T}\left[a\left(s, z_{\epsilon}(s)\right)-\bar{a}\left(z_{\epsilon}(s)\right)\right] d s\right|^{2} \\
& +12 \epsilon^{4 H} E\left|\int_{m T}^{t}\left[a\left(s, z_{\epsilon}(s)\right)-\bar{a}\left(z_{\epsilon}(s)\right)\right] d s\right|^{2} \\
\leq & 36 \epsilon^{4 H} E\left|\sum_{i=1}^{m} \int_{(i-1) T}^{i T}\left[a\left(s, z_{\epsilon}(s)\right)-a\left(s, z_{\epsilon}(i T)\right)\right] d s\right|^{2} \\
& +36 \epsilon^{4 H} E\left|\sum_{i=1}^{m} \int_{(i-1) T}^{i T}\left[a\left(s, z_{\epsilon}(i T)\right)-\bar{a}\left(z_{\epsilon}(i T)\right)\right] d s\right|^{2} \\
& +36 \epsilon^{4 H} E\left|\sum_{i=1}^{m} \int_{(i-1) T}^{i T}\left[\bar{a}\left(z_{\epsilon}(i T)\right)-\bar{a}\left(z_{\epsilon}(s)\right)\right] d s\right|^{2} \\
& +12 \epsilon^{4 H} E\left|\int_{m T}^{t}\left[a\left(s, z_{\epsilon}(s)\right)-\bar{a}\left(z_{\epsilon}(s)\right)\right] d s\right|^{2} .
\end{aligned}
$$

Note that, by the definition of $\bar{a}$, we have

$$
\begin{aligned}
& E\left|\sum_{i=1}^{m} \int_{(i-1) T}^{i T}\left[a\left(s, z_{\epsilon}(i T)\right)-\bar{a}\left(z_{\epsilon}(i T)\right)\right] d s\right|^{2} \\
& \quad \leq m \sum_{i=1}^{m} E\left|\int_{0}^{T} a\left(s, z_{\epsilon}(i T)\right) d s-T \bar{a}\left(z_{\epsilon}(i T)\right)\right|^{2} \\
& \quad=0
\end{aligned}
$$

thus, by the Jensen inequality and Assumptions A, C, Eq. (6) becomes

$$
Q_{12} \leq 72 \epsilon^{4 H} m T \int_{0}^{T} \kappa\left(E\left|z_{\epsilon}(s)-z_{\epsilon}(i T)\right|^{2}\right) d s+48 \epsilon^{4 H} u M T .
$$

Then we can deduce that $Q_{1}$ has the following approximation:

$$
\begin{aligned}
Q_{1} \leq & 6 \epsilon^{4 H} u \int_{0}^{t} \kappa\left(E\left|x_{\epsilon}(s)-z_{\epsilon}(s)\right|^{2}\right) d s \\
& +72 \epsilon^{4 H} m T \int_{0}^{T} \kappa\left(E\left|z_{\epsilon}(s)-z_{\epsilon}(i T)\right|^{2}\right) d s+48 \epsilon^{4 H} u M T \\
:= & \epsilon^{H} K_{1} \int_{0}^{t} \kappa\left(E\left|x_{\epsilon}(s)-z_{\epsilon}(s)\right|^{2}\right) d s \\
& +\epsilon^{H} K_{2} \int_{0}^{T} \kappa\left(E\left|z_{\epsilon}(s)-z_{\epsilon}(i T)\right|^{2}\right) d s+\epsilon^{H} O_{1} .
\end{aligned}
$$


Now, to estimate $Q_{2}$, we have

$$
\begin{aligned}
Q_{2} \leq & 6 \epsilon^{2 H} E\left|\int_{0}^{t} b\left(s, x_{\epsilon}(s)\right)-b\left(s, z_{\epsilon}(s)\right) d^{\circ} W^{H}(s)\right|^{2} \\
& +6 \epsilon^{2 H} E\left|\int_{0}^{t} b\left(s, z_{\epsilon}(s)\right)-\bar{b}\left(z_{\epsilon}(s)\right) d^{\circ} W^{H}(s)\right|^{2} \\
= & Q_{21}+Q_{22} .
\end{aligned}
$$

Thanks to Lemma 2.7 and Assumption A, we can obtain

$$
Q_{21} \leq 12 \epsilon^{2 H} u^{2 H-1} H \int_{0}^{t} \kappa\left(E\left|x_{\epsilon}(s)-z_{\epsilon}(s)\right|^{2}\right) d s+24 \epsilon^{2 H} u^{2} C
$$

And, similar to Eq. (6),

$$
\begin{aligned}
Q_{22} \leq & 12 \epsilon^{2 H} E\left|\sum_{i=1}^{m} \int_{(i-1) T}^{i T}\left[b\left(s, z_{\epsilon}(s)\right)-\bar{b}\left(z_{\epsilon}(s)\right)\right] d^{\circ} W^{H}(s)\right|^{2} \\
& +12 \epsilon^{2 H} E\left|\int_{m T}^{t}\left[b\left(s, z_{\epsilon}(s)\right)-\bar{b}\left(z_{\epsilon}(s)\right)\right] d^{\circ} W^{H}(s)\right|^{2} \\
\leq & 36 \epsilon^{2 H} E\left|\sum_{i=1}^{m} \int_{(i-1) T}^{i T}\left[b\left(s, z_{\epsilon}(s)\right)-b\left(s, z_{\epsilon}(i T)\right)\right] d^{\circ} W^{H}(s)\right|^{2} \\
& +36 \epsilon^{2 H} E\left|\sum_{i=1}^{m} \int_{(i-1) T}^{i T}\left[b\left(s, z_{\epsilon}(i T)\right)-\bar{b}\left(z_{\epsilon}(i T)\right)\right] d^{\circ} W^{H}(s)\right|^{2} \\
& +36 \epsilon^{2 H} E\left|\sum_{i=1}^{m} \int_{(i-1) T}^{i T}\left[\bar{b}\left(z_{\epsilon}(i T)\right)-\bar{b}\left(z_{\epsilon}(s)\right)\right] d^{\circ} W^{H}(s)\right|^{2} \\
& +12 \epsilon^{2 H} E\left|\int_{m T}^{t}\left[b\left(s, z_{\epsilon}(s)\right)-\bar{b}\left(z_{\epsilon}(s)\right)\right] d^{\circ} W^{H}(s)\right|^{2}
\end{aligned}
$$

employing Lemma 2.7 and Assumptions A, C implies

$$
\begin{aligned}
Q_{22} \leq & 144 \epsilon^{2 H} u^{2 H-1} m T H \int_{0}^{T} \kappa\left(E\left|z_{\epsilon}(s)-z_{\epsilon}(i T)\right|^{2}\right) d s+288 \epsilon^{2 H} C u^{2} \\
& +72 \epsilon^{2 H} u^{2 H-1} m T H E \int_{0}^{T}\left|b\left(s, z_{\epsilon}(s)\right)-\bar{b}\left(z_{\epsilon}(s)\right)\right|^{2} d s+144 \epsilon^{2 H} C u^{2} \\
& +24 \epsilon^{2 H} u^{2 H-1} H E \int_{0}^{T}\left|b\left(s, z_{\epsilon}(s)\right)-\bar{b}\left(z_{\epsilon}(s)\right)\right|^{2} d s+48 \epsilon^{2 H} C u^{2} \\
\leq & 144 \epsilon^{2 H} u^{2 H-1} m T H \int_{0}^{T} \kappa\left(E\left|z_{\epsilon}(s)-z_{\epsilon}(i T)\right|^{2}\right) d s+288 \epsilon^{2 H} C u^{2} \\
& +288 \epsilon^{2 H} u^{2 H-1} m H T^{2} M+144 \epsilon^{2 H} C u^{2} \\
& +96 \epsilon^{2 H} u^{2 H-1} m H T^{2} M+48 \epsilon^{2 H} C u^{2} .
\end{aligned}
$$


Consequently, taking $Q_{21}$ and $Q_{22}$ into account, we conclude that

$$
\begin{aligned}
Q_{2} \leq & 12 \epsilon^{2 H} u^{2 H-1} H \int_{0}^{t} \kappa\left(E\left|x_{\epsilon}(s)-z_{\epsilon}(s)\right|^{2}\right) d s+24 \epsilon^{2 H} u^{2} C \\
& +144 \epsilon^{2 H} u^{2 H-1} m T H \int_{0}^{T} \kappa\left(E\left|z_{\epsilon}(s)-z_{\epsilon}(i T)\right|^{2}\right) d s+288 \epsilon^{2 H} C u^{2} \\
& +288 \epsilon^{2 H} u^{2 H-1} m H T^{2} M+144 \epsilon^{2 H} C u^{2}+96 \epsilon^{2 H} u^{2 H-1} m H T^{2} M+48 \epsilon^{2 H} C u^{2} \\
:= & \epsilon^{H} K_{3} \int_{0}^{t} \kappa\left(E\left|x_{\epsilon}(s)-z_{\epsilon}(s)\right|^{2}\right) d s \\
& +\epsilon^{H} K_{4} \int_{0}^{T} \kappa\left(E\left|z_{\epsilon}(s)-z_{\epsilon}(i T)\right|^{2}\right) d s+\epsilon^{H} O_{2} .
\end{aligned}
$$

Arriving at the last term $Q_{3}$, we apply Assumption B to obtain

$$
\begin{aligned}
Q_{3} \leq & 6 \epsilon^{4 H} k(m+1) E \sum_{j=1}^{k}\left|I_{j}\left(x_{\epsilon}\left(t_{j}\right)\right)\right|^{2} \\
& +6 \epsilon^{4 H} k(m+1) \frac{t}{T^{2}} E \sum_{j=1}^{k} \int_{0}^{t}\left|I_{j}\left(z_{\epsilon}(s)\right)\right|^{2} d s \\
\leq & 6 \epsilon^{4 H} k^{2}(m+1) N_{1}+6 \epsilon^{4 H} k^{2}(m+1)^{2} N_{1}:=\epsilon^{H} O_{3} .
\end{aligned}
$$

Now, combining (7), (9), and (10) together, we get

$$
\begin{aligned}
E\left|x_{\epsilon}(t)-z_{\epsilon}(t)\right|^{2} \leq & \epsilon^{H} \tilde{O}+\epsilon^{H}\left(K_{2}+K_{4}\right) \int_{0}^{T} \kappa\left(E\left|z_{\epsilon}(s)-z_{\epsilon}(i T)\right|^{2}\right) d s \\
& +\epsilon^{H}\left(K_{1}+K_{3}\right) \int_{0}^{t} \kappa\left(E\left|x_{\epsilon}(s)-z_{\epsilon}(s)\right|^{2}\right) d s,
\end{aligned}
$$

where $\tilde{O}=O_{1}+O_{2}+O_{3}$. Obviously, the function $\kappa(x)$ is nondecreasing on $\mathbb{R}_{+}$and $\kappa(0)=0$. Then, for any $t_{0}>0$, by setting $G(t)=\int_{t_{0}}^{t} \frac{d s}{\kappa(s)}$, it follows from Lemma 2.8 that

$$
\begin{aligned}
E\left|x_{\epsilon}(t)-z_{\epsilon}(t)\right|^{2} \leq & G^{-1}\left(G\left[\epsilon^{H} \tilde{O}+\epsilon^{H}\left(K_{2}+K_{4}\right) \int_{0}^{T} \kappa\left(E\left|z_{\epsilon}(s)-z_{\epsilon}(i T)\right|^{2}\right) d s\right]\right. \\
& \left.+\epsilon^{H}\left(K_{1}+K_{3}\right) T\right) .
\end{aligned}
$$

Note that

$$
\left\{\epsilon^{H} \tilde{O}+\epsilon^{H}\left(K_{2}+K_{4}\right) \int_{0}^{T} \kappa\left(E\left|z_{\epsilon}(s)-z_{\epsilon}(i T)\right|^{2}\right) d s\right\} \rightarrow 0
$$

as $\epsilon$ converges to zero. Recalling the condition $\int_{0^{+}} \frac{d s}{\kappa(s)}=\infty$, we can conclude that

$$
G\left[\epsilon^{H} \tilde{O}+\epsilon^{H}\left(K_{2}+K_{4}\right) \int_{0}^{T} \kappa\left(E\left|z_{\epsilon}(s)-z_{\epsilon}(i T)\right|^{2}\right) d s\right]+\epsilon^{H}\left(K_{1}+K_{3}\right) T \rightarrow-\infty .
$$


On the other hand, because the function $G$ is strictly increasing, we obtain that $G$ has an inverse function which is strictly increasing too, and $G^{-1}(-\infty)=0$. Namely,

$$
G^{-1}\left(G\left[\epsilon^{H} \tilde{O}+\epsilon^{H}\left(K_{2}+K_{4}\right) \int_{0}^{T} \kappa\left(E\left|z_{\epsilon}(s)-z_{\epsilon}(i T)\right|^{2}\right) d s\right]+\epsilon^{H}\left(K_{1}+K_{3}\right) T\right) \rightarrow 0
$$

as $\epsilon \rightarrow 0$. Finally, we get

$$
\begin{array}{rl}
\lim _{\epsilon \rightarrow 0} & E\left|x_{\epsilon}(t)-z_{\epsilon}(t)\right|^{2} \\
\leq & \lim _{\epsilon \rightarrow 0}\left(G ^ { - 1 } \left(G\left[\epsilon^{H}(\tilde{O})+\epsilon^{H}\left(K_{2}+K_{4}\right) \int_{0}^{T} \kappa\left(E\left|z_{\epsilon}(s)-z_{\epsilon}(i T)\right|^{2}\right) d s\right]\right.\right. \\
& \left.\left.+\epsilon^{H}\left(K_{1}+K_{3}\right) T\right)\right) \\
= & 0 .
\end{array}
$$

This completes the proof.

Remark 3.2 In Theorem 3.1, we establish the strong convergence (in the moment sense) of the processes $x_{\epsilon}$ and $z_{\epsilon}$ under non-Lipschitz condition. In other words, we have proved that, for a sufficiently small $\epsilon$, the solutions of $x_{\epsilon}$ and $z_{\epsilon}$ are close enough.

For the sake of establishing the periodic stochastic averaging of Eq. (4) in finite time interval, we need the following auxiliary lemma.

Lemma 3.3 Let (4) be averaged SDE of standard ISDE (3). If Assumption C holds, then, for $\epsilon_{1} \in\left(0, \epsilon_{0}\right]$, there exist $\epsilon \in\left(0, \epsilon_{1}\right]$ and a positive constant $D>0$ such that

$$
E\left|z_{\epsilon}(t)-z_{\epsilon}(i T)\right|^{2} \leq D
$$

for all $t \in[(i-1) T, i T], i=1,2, \ldots, m, m \in \mathbb{N}$.

Proof By Eq. (4), taking expectation and using the simple inequality $|a+b|^{2} \leq 2|a|^{2}+2|b|^{2}$ yield

$$
E\left|z_{\epsilon}(t)-z_{\epsilon}(i T)\right|^{2} \leq 2 \epsilon^{4 H} E\left|\int_{i T}^{t} \bar{a}\left(z_{\epsilon}(s)\right) d s\right|^{2}+2 \epsilon^{2 H} E\left|\int_{i T}^{t} \bar{b}\left(z_{\epsilon}(s)\right) d^{\circ} W^{H}(s)\right|^{2} .
$$

Now, let $0 \leq t \leq u \leq T$, then by the Cauchy-Schwarz inequality, Lemma 2.7, and Assumption $\mathrm{C}$ we get

$$
E\left|\int_{i T}^{t} \bar{a}\left(z_{\epsilon}(s)\right) d s\right|^{2} \leq u E \int_{i T}^{t}\left|\bar{a}\left(z_{\epsilon}(s)\right)\right|^{2} d s \leq(m+1) T^{2} M
$$

and

$$
\begin{aligned}
E\left|\int_{i T}^{t} \bar{b}\left(z_{\epsilon}(s)\right) d^{\circ} W^{H}(s)\right|^{2} & \leq 2 H u^{2 H-1} E \int_{i T}^{t}\left|\bar{b}\left(z_{\epsilon}(s)\right)\right|^{2} d s+4 C u^{2} \\
& \leq 2 H(m+1)^{2 H-1} T^{2 H} M+4 C(m+1)^{2} T^{2}
\end{aligned}
$$

where $m$ is the largest integer such that $m T \leq t$. 
Finally, one can deduce that

$$
\begin{aligned}
E\left|z_{\epsilon}(t)-z_{\epsilon}(i T)\right|^{2} \leq & 2 \epsilon^{4 H}(m+1) T^{2} M+4 \epsilon^{2 H} H(m+1)^{2 H-1} T^{2 H} M \\
& +8 \epsilon^{2 H} C(m+1)^{2} T^{2} \\
:= & D .
\end{aligned}
$$

Hence, proved.

Theorem 3.4 Suppose that Assumptions A-C are fulfilled for standard ISDE (3) and for averaged $S D E$ (4), then, for given $\beta>0, \alpha \in(0,1)$, and $\epsilon_{1} \in\left(0, \epsilon_{0}\right]$, there exist $\gamma>0$ and $\epsilon \in\left(0, \epsilon_{1}\right]$ such that

$$
E\left|x_{\epsilon}(t)-z_{\epsilon}(t)\right|^{2} \leq \gamma \epsilon^{H}
$$

for all $t \in\left[0, \beta \epsilon^{-\alpha H}\right]$.

Proof By Assumption A, the concave function $\kappa$ satisfies

$$
\kappa(x) \leq \lambda_{1} x+\lambda_{2}
$$

where $\lambda_{1}$ and $\lambda_{2}$ are positive constants. Applying this property for Eq. (11) yields

$$
\begin{aligned}
E\left|x_{\epsilon}(t)-z_{\epsilon}(t)\right|^{2} \leq & \epsilon^{H} K_{5} \lambda_{1} \int_{0}^{t} E\left|x_{\epsilon}(s)-z_{\epsilon}(s)\right|^{2} d s+\epsilon^{H} K_{5} \lambda_{2} t \\
& +\epsilon^{H} K_{6} \lambda_{1} \int_{0}^{T} E\left|z_{\epsilon}(s)-z_{\epsilon}(i T)\right|^{2} d s+\epsilon^{H} K_{6} \lambda_{2} T+\epsilon^{H} \tilde{O} .
\end{aligned}
$$

Thanks to Lemma 3.3, we can obtain

$$
\begin{aligned}
E\left|x_{\epsilon}(t)-z_{\epsilon}(t)\right|^{2} \leq & \epsilon^{H} K_{5} \lambda_{1} \int_{0}^{t} E\left|x_{\epsilon}(s)-z_{\epsilon}(s)\right|^{2} d s+\epsilon^{H} K_{5} \lambda_{2} t \\
& +\epsilon^{H} K_{6} \lambda_{1} D T+\epsilon^{H} K_{6} \lambda_{2} T+\epsilon^{H} \tilde{O} \\
:= & \epsilon^{H} K \int_{0}^{t} E\left|x_{\epsilon}(s)-z_{\epsilon}(s)\right|^{2} d s+\epsilon^{H} O
\end{aligned}
$$

Finally, applying Gronwall's inequality implies

$$
E\left|x_{\epsilon}(t)-z_{\epsilon}(t)\right|^{2} \leq \epsilon^{H} O e^{\epsilon^{H} K t} .
$$

Now, choose $\alpha \in(0,1)$ and $\beta>0$, we can select $\epsilon_{1} \in\left(0, \epsilon_{0}\right]$ such that, for every $\epsilon \in\left(0, \epsilon_{1}\right]$, $t \in\left[0, \beta \epsilon^{-\alpha H}\right] \subseteq[0, \infty)$. And let $\gamma=O e^{K \beta}$, we conclude

$$
E\left|x_{\epsilon}(t)-z_{\epsilon}(t)\right|^{2} \leq \gamma \epsilon^{H}
$$

Therefore, Theorem 3.4 is proved. 
Remark 3.5 Theorem 3.4 indicates that the order of convergence of the processes $x_{\epsilon}$ and $z_{\epsilon}$ in finite time is about $\epsilon^{-\alpha H}$ for $\alpha \in(0,1)$.

Next, we shall use the previous results to establish the convergence in probability between the solutions of Eq. (3) and Eq. (4).

Corollary 3.6 Let Assumptions A-C hold, for arbitrary small number $\delta>0$, there exist $\epsilon_{1} \in\left(0, \epsilon_{0}\right], \beta>0$, and $0<\alpha<1$ such that, for all $\epsilon \in\left(0, \epsilon_{1}\right]$, we have

$$
\lim _{\epsilon \rightarrow 0} P\left(\sup _{0 \leq t \leq \beta \epsilon^{-\alpha H}}\left|x_{\epsilon}(t)-z_{\epsilon}(t)\right|>\delta\right)=0 .
$$

Proof By Theorem 3.4 and employing the Chebyshev-Markov inequality, for any given number $\delta>0$, one can obtain that

$$
\begin{aligned}
P\left(\sup _{0 \leq t \leq \beta \epsilon^{-\alpha H}}\left|x_{\epsilon}(t)-z_{\epsilon}(t)\right|>\delta\right) & \leq \frac{1}{\delta^{2}} E\left(\sup _{0 \leq t \leq \beta \epsilon^{-\alpha H}}\left|x_{\epsilon}(t)-z_{\epsilon}(t)\right|^{2}\right) \\
& \leq \frac{\epsilon^{H} O e^{\epsilon^{H} K t}}{\delta^{2}}
\end{aligned}
$$

letting $\epsilon \rightarrow 0$. Then the required result follows.

\section{Example}

In this section, we provide an example to illustrate the foregoing averaging principle results.

Consider the following impulsive stochastic dynamical system:

$$
\begin{aligned}
& d x_{\epsilon}(t)=-\epsilon^{2 H} d t+\epsilon^{H} \cos ^{2}(t) \lambda d^{\circ} W^{H}(t), \quad t \neq t_{j} \\
& \triangle x_{\epsilon}(t)=\epsilon^{2 H} j^{3} x_{\epsilon}\left(t_{j}^{-}\right), \quad t=t_{j}, j \in \mathbb{N}, \\
& x_{\epsilon}(0)=x_{0},
\end{aligned}
$$

where $a(t, x)=-1, b(t, x)=\lambda \cos ^{2}(t)$, and $I_{j}(x)=j^{3} x$. Let $T=1$ and $\lambda=3$. Then, by the definitions of $\bar{a}(\cdot), \bar{b}(\cdot)$, and $\bar{I}(\cdot)$ in Sect. 2, we have

$$
\begin{aligned}
& \bar{a}\left(z_{\epsilon}\right)=\frac{1}{T} \int_{0}^{T} a\left(t, z_{\epsilon}\right) d t=-1 \\
& \bar{b}\left(z_{\epsilon}\right)=\frac{1}{T} \int_{0}^{T} b\left(t, z_{\epsilon}\right) d t=\lambda \int_{0}^{1} \cos ^{2}(t) d t=3 \times 0.73=2.19 \\
& \bar{I}\left(z_{\epsilon}\right)=\frac{1}{T} \sum_{j=1}^{k} j^{3} z_{\epsilon}=z_{\epsilon} \sum_{j=1}^{k} j^{3}=\frac{k^{2}(k+1)^{2}}{4} z_{\epsilon} .
\end{aligned}
$$

So then, the solution to averaged SDE for the impulsive dynamical system (13) can be interpreted as follows:

$$
z_{\epsilon}(t)=x_{0}+\frac{k^{2}(k+1)^{2}}{4} \epsilon^{2 H} \int_{0}^{t}\left(z_{\epsilon}(s)-1\right) d s+2.19 \epsilon^{H} \int_{0}^{t} d^{\circ} W^{H}(t) .
$$


It is easy to verify that the conditions of Theorem 3.1, Theorem 3.4, and Corollary 3.6 are satisfied. Then the solution of averaged SDEs (14) converges to that of standard Eq. (13) in the sense of mean square and in probability.

\section{Acknowledgements}

The authors would like to thanks the editors and the referees for their helpful comments and suggestions.

\section{Funding}

This work is partly supported by the National Natural Science Foundation of China under Grant (11531006).

Availability of data and materials

Not applicable.

\section{Competing interests}

The authors declare that there is no conflict of interests.

\section{Authors' contributions}

All authors contributed equally to the writing of this paper. All authors read and approved the final manuscript.

\section{Author details}

'School of Mathematics and Statistics, Huazhong University of Science and Technology, Wuhan, P.R. China. ${ }^{2}$ Department of Mathematical Statistics, Faculty of Graduate Studies for Statistical Research, Cairo University, Giza, Egypt.

\section{Publisher's Note}

Springer Nature remains neutral with regard to jurisdictional claims in published maps and institutional affiliations.

Received: 16 September 2019 Accepted: 12 December 2019 Published online: 18 December 2019

\section{References}

1. Mandelbrot, B.B., Van Ness, J.W.: Fractional Brownian motions, fractional noises and applications. SIAM Rev. 10(4), 422-437 (1968)

2. Dai, W., Heyde, C.C.: Ito's formula with respect to fractional Brownian motion and its application. J. Appl. Math. Stoch. Anal. 9(4), 439-448 (1996)

3. Chakravarti, N., Sebastiar, K.L.: Fractional Brownian motion models for polymers. Chem. Phys. Lett. 267(1-2), 9-13 (1997)

4. Scheffer, R., Maciel, F.R.: The fractional Brownian motion as a model for an industrial airlift reactor. Chem. Eng. Sci. 56(2), 707-711 (2001)

5. Hernandez, E., Keck, D.N., McKibben, M.A.: On a class of measure-dependent stochastic evolution equations driven by fBm. J. Appl. Math. Stoch. Anal. 26, Article ID 69747 (2007)

6. Biagini, F., Hu, Y., Øksendal, B., Zhang, T.: Stochastic Calculus for Fractional Brownian Motion and Applications. Springer, Berlin (2008)

7. Samoilenko, A.M., Perestyuk, N.A.: Impulsive Differential Equations. World Scientific, Singapore (1955)

8. Catllá, A.J., Schaeffer, D.G., Witelski, T.P., Monson, E.E., Lin, A.L.: On spiking models for synaptic activity and impulsive differential equations. SIAM Rev. 50(3), 553-569 (2008)

9. Li, X.D., Bohner, M., Wang, C.K.: Impulsive differential equations: periodic solutions and applications. Automatica 52, $173-178$ (2015)

10. Girel, S., Crauste, F.: Existence and stability of periodic solutions of an impulsive differential equation and application to CD8 T-cell differentiation. J. Math. Biol. 76(7), 1765-1795 (2018)

11. Krylov, N.M., Bogolyubov, N.N.: Les proprietes ergodiques des suites des probabilites enchaine. C. R. Math. Acad. Sci. 204, 1454-1546 (1937)

12. Volosov, V.M.: Averaging in systems of ordinary differential equations. Russ. Math. Surv. 17, 1-126 (1962)

13. Khasminskii, R.Z: On the principle of averaging the Itô stochastic differential equations. Kibernetika 4, 260-279 (1968)

14. Veretennikov, A.Y: On the averaging principle for systems of stochastic differential equations. Math. USSR Sb. 69(1), 271-284 (1991)

15. Hale, J.K.: Averaging methods for differential equations with retarded arguments with a small parameter. J. Differ. Equ. 2(1), 57-73 (1966)

16. Federson, M., Mesquita, J.G.: Non-periodic averaging principles for measure functional differential equations and functional dynamic equations on time scales involving impulses. J. Differ. Equ. 255(10), 3098-3126 (2013)

17. Stratonovic, R.L.: Topics in the Theory of Random Noise. Mathematics and Its Applications. Gordon \& Breach, New York (1963)

18. Freidlin, M.I., Wentzell, A.D.: Random perturbations. In: Random Perturbations of Dynamical Systems, pp. 15-43. Springer, Berlin (1998)

19. Abouagwa, M., Li, J.: Approximation properties of solutions to Itô-Doob stochastic fractional differential equations with non-Lipschitz coefficients. Stoch. Dyn. 19, Article ID 1950029 (2019)

20. Stoyanov, I., Bainov, D.: The averaging method for a class of stochastic differential equations. Ukr. Math. J. 26, 186-194 (1974)

21. Kolomiets, V.G., Mel'nikov, A.I: Averaging of stochastic systems of integral-differential equations with Poisson noise. Ukr. Math. J. 43(2), 242-246 (1991) 
22. Xu, Y., Duan, J., Xu, W.: An averaging principle for stochastic dynamical systems with Lévy noise. Physica D 240(17), 1395-1401 (2011)

23. Abouagwa, M., Li, J: Stochastic fractional differential equations driven by Lévy noise under Carathéodory conditions. J. Math. Phys. 60(2), Article ID 022701 (2019)

24. Ma, S., Kang, Y.: Periodic averaging method for impulsive stochastic differential equations with Lévy noise. Appl. Math. Lett. 93, 91-97 (2019)

25. Xu, Y., Pei, B., Li, Y.: Approximation properties for solutions to non-Lipschitz stochastic differential equations with Lévy noise. Math. Methods Appl. Sci. 38, 2120-2131 (2015)

26. Abouagwa, M., Li, J.: G-neutral stochastic differential equations with variable delay and non-Lipschitz coefficients. Discrete Contin. Dyn. Syst., Ser. B (2019). https://doi.org/10.3934/dcdsb.2019241

27. He, X.Y., Han, S., Tao, J.: Averaging principle for SDEs of neutral type driven by G-Brownian motion. Stoch. Dyn. 19 Article ID 1950004 (2019)

28. Xu, Y., Guo, R., Liu, D., Zhang, H., Duan, J.: Stochastic averaging for dynamical systems with fractional Brownian motion. Discrete Contin. Dyn. Syst., Ser. B 19(4), 1197-1212 (2014)

29. Xu, Y., Pei, B., Wu, J.L.: Stochastic averaging principle for differential equations with non-Lipschitz coefficients driven by fractional Brownian motion. Stoch. Dyn. 17(2), Article ID 1750013 (2017)

30. Xu, Y., Pei, B., Guo, R.: Stochastic averaging for slow-fast dynamical systems with fractional Brownian motion. Discrete Contin. Dyn. Syst., Ser. B 20(7), 2257-2267 (2015)

31. Pei, B., Xu, Y., Wu, J.L.: Stochastic averaging for stochastic differential equations driven by fractional Brownian motion and standard Brownian motion. Appl. Math. Lett. 100, Article ID 106006 (2020)

32. Russo, F., Vallois, P.: Forward, backward and symmetric stochastic integration. Probab. Theory Relat. Fields 97(3), 403-421 (1993)

33. Feyel, D., de la Pradelle, A.: On fractional Brownian processes. Potential Anal. 10(3), 273-288 (1999)

34. Duncan, T.E., Hu, Y., Pasik-Duncan, B.: Stochastic calculus for fractional Brownian motion I. Theory. SIAM J. Control Optim. 38(2), 582-612 (2000)

35. Alòs, E., Nualart, D.: Stochastic integration with respect to the fractional Brownian motion. Stoch. Stoch. Rep. 75(3), 129-152 (2003)

36. Bihari, I.: A generalization of a lemma of Bellman and its application to uniqueness problem of differential equations. Acta Math. Acad. Sci. Hung. 7(1), 81-94 (1956)

37. Shen, L.J., Sun, J.T.: Global existence of solutions for stochastic impulsive differential equations. Acta Math. Sin. 27(4), 773-780 (2011)

38. Abouagwa, M., Cheng, F.F., Li, J.: Impulsive stochastic fractional differential equations driven by fractional Brownian motion. Adv. Differ. Equ. (under review)

39. Mao, W., Hu, L.J., You, S.R., Mao, X.R.: The averaging method for multivalued SDEs with jumps and non-Lipschitz coefficients. Discrete Contin. Dyn. Syst., Ser. B 24(9), 4937-4954 (2019)

\section{Submit your manuscript to a SpringerOpen ${ }^{\circ}$ journal and benefit from:}

- Convenient online submission

- Rigorous peer review

- Open access: articles freely available online

- High visibility within the field

- Retaining the copyright to your article

Submit your next manuscript at $\boldsymbol{\nabla}$ springeropen.com 